\title{
Hemiarthroplasty or Total Hip Replacement for intracapsular Hip Fractures? A Dilemma in Trauma Surgery
}

\author{
Rhys Morris ${ }^{1}$, Michael H Lewis², Damian M Bailey²* and Peter M Lewis ${ }^{3}$ \\ ${ }^{1}$ Robert Jones and Agnes Hunt Orthopaedic Hospital, Oswestry, Shropshire, SY10 7AG, UK \\ ${ }^{2}$ Neurovascular Research Laboratory, Faculty of Life Sciences and Education, University of South Wales, UK \\ ${ }^{3}$ Cwm Taf Morgannwg University Health Board, Prince Charles \& Royal Glamorgan Hospitals, South Wales, UK \\ *Corresponding author: Damian M Bailey, Neurovascular Research Laboratory, Faculty of Life Sciences and Education, University of South Wales
}

Received: September 06, 2019; Accepted: September 25, 2019; Published: October 01, 2019;

Hip fractures in the elderly are a common and devastating injury, placing a considerable burden on healthcare systems around the world. In the UK there are over 70,000 hip fractures annually, costing around $£ 2$ billion [1]. Given the ever-ageing population, future estimates suggest that that over 6 million hip fractures/year will occur worldwide by 2050 [2]. Mortality and morbidity following these injuries remains high, in England with a 30-day mortality of 8.5\% [3].

Displaced intracapsular fractures are at risk of non-union and avascular necrosis, and treatment in the form of a hemiarthroplasty or Total Hip Replacement (THR) is recommended [4]. The choice between these remains controversial [5], with potential benefits and risks associated with each. Traditionally, hemiarthroplasty has been the mainstay of treatment as it is less complex and thus quicker surgery, with reduced bleeding and complications [6]. However, some studies suggest improved function following a THR [7], and surgeons worry about long-term acetabular wear from hemiarthroplasties, and the subsequent need for conversion to a THR [8].

Population studies in the USA [9], Finland [10] and South Korea [11] have shown trends demonstrating increasing utilisation of THR in these patients for this fracture. In the UK, in 2011, the National Institute of Health and Clinical Excellence (NICE) produced guidance on when a THR should be offered to hip fracture patients [12]. They recommended offering a THR to patients who: (a) could walk independently, (b) were not cognitively impaired, and (c) were medically fit for anaesthesia and the procedure [12]. By 2017 the first of these criteria was revised to patients who are able to walk independently outdoors with no more than the use of a stick [13]. Despite this, in the UK compliance to NICE guidelines remains poor, with one study, published in 2016, of over 100,000 patients showing less than a third of eligible patients received a THR [4].

Several potential reasons exist regarding this low compliance. First, these cases require an experienced arthroplasty surgeon [4], not always feasible especially in smaller centres, contributing to a delay in treatment, and increased morbidity and mortality. In our unit, we have shown in an as of yet unpublished retrospective study of patients who all met the NICE criteria that those receiving a THR waited considerably longer than hemiarthroplasty patients (3.7 days versus 1 day respectively, $P<0.05)$. Second, it has been acknowledged the precise indications for THRs in hip fractures are not well defined [4] with some authors feeling the current NICE criteria are too inclusive [14], particularly in patients with significant co-morbidities (the most common reason hemiarthroplasties were chosen over THRs) [14]. This was supported as hemiarthroplasty patients were older, and had significantly increased 1 year mortality, suggesting greater frailty in these patients, despite all being eligible for THRs [14]. In our local study we too found those undergoing a hemiarthroplasty were older (mean age 83 vs 73 years) and had an increased 1 year mortality (18.2\% vs $8.3 \%)$, despite all patients meeting NICE criteria. Indeed, one population-based study on THR usage in hip fractures showed NICE guidance was less likely to be followed in older patients, and those with worse cognition, ASA grade and ambulatory status [4].

The literature on the outcome of THRs compared to hemiarthroplasties is also equivocal, with a variety of studies supporting each approach. One recent meta-analysis of prospective studies supported THR [15], demonstrating improvements in function as measured by the Harris Hip Score (HHS) and Quality of Life (SF-36), reduced re-operation rates [15] and beyond 4 years no difference in dislocation rates [15]. However, the authors acknowledge inconsistencies in trial design [15], and it is worth noting the implants and selection criteria varied widely between studies. Interestingly, the authors also conclude those patients older than 80 years, or those with a short life expectancy, both THR and hemiarthroplasty are both reasonable interventions [15].

Another retrospective UK study using over 7,000 matched patients, on a national database, showed no difference in revision rates between implants [16]. This finding was reinforced by another study showing the conversion rate of hemiarthroplasties to THRs for acetabular wear was low, particularly in older patients $(1.4 \%$ in patients older than 75 years) [8].

The short to medium term dislocation rate in THR patients has been shown to be significantly higher than for hemiarthroplasty patients $[16,17]$. A randomised prospective study assessing longterm outcomes at 12 years found no difference in complication or reoperation rates between groups, and actually demonstrated equivalent function as measured using the modified HHS [5]. This study concluded by advising cemented hemiarthroplasty in hip fracture 
patients aged greater than 70 years, in the absence of radiological evidence of joint degeneration [5].

In conclusion, THR surgery was once famously described as the 'operation of the century [18], helping to revolutionise the management of patients crippled with osteoarthritis [18]. Its role in these patients is not disputed. However its role in trauma remains controversial [5]. We feel THR can also achieve excellent results in hip fracture patients, but at present the ideal patient, and precise indications are not well defined [4]. Furthermore emergency surgery is usually defined 'as life or limb saving' which should be as simple and expeditious as possible, particularly in the elderly and infirm. The decision for THR or hemiarthroplasty is multi-factorial and includes surgical experience, facilities and importantly patient morbidity/ASA, frailty and age. It is our opinion that current NICE guidelines are too inclusive. Until more conclusive data shows otherwise, surgical decision-making should remain at the discretion of the attending surgical team and local circumstances.

\section{References}

1. Royal College of Physicians (2014) National Hip Fracture Database annual report London

2. Dhanwal DK, Dennison EM, Harvey NC (2011) Epidemiology of hip fracture: worldwide geographic variation. Indian J Orthop 15-22.

3. Neuburger J, Currie C, Wakeman R (2015) The impact of a national clinician-led audit initiative on care and mortality after hip fracture in England: an external evaluation using time trends in non-audit data. Med Care 686-91.

4. Perry DC, Metcalfe D, Griffin XL (2016) Inequalities in use of total hip arthroplasty for hip fracture: population based study. $B M J$

5. Tol CJM, van den Bekerom MPJ, Sieneveldt (2017) Hemiarthroplasty or total hip arthroplasty for the treatment of a displaced intracapsular fracture in active elderly patients. 12-year follows up of randomised trial. Bone Joint J 250-54

6. Keating J, Grant A, Masson M (2005) Displaced intracapsular hip fractures in fir, older people: a randomised comparison of reduction and fixation, bipolar hemiarthroplasty and total hip arthroplasty. Health Technol Assess 1-65.

7. Avery PP, Baker RP, Walton MJ (2011) Total hip replacement and hemiarthroplasty in mobile, independent patients with a displaced intracapsular fracture of the femoral neck: a seven-to ten-year follow-up report of a prospective randomised controlled trial. J Bone Joint Surg Br 93: 1045-8.

8. Grosso MJ, Danoff JR, Murtagh JS (2017) Hemiarthroplasty for displaced femoral neck fractures in the elderly has a low conversion rate. J Arthroplasty 32: 150-54

9. Bishop J, Yang A, Githens M (2016) Evaluation of contemporary trends in femoral neck fracture management reveals discrepancies in treatment. Geriatr Orthop Surg Rehabil 7:135-41.

10. Hongisto MT, Pihlajamaki H, Niemi S (2014) Surgical procedures in femoral neck fractures in Finland: a nationwide study between 1998 and 2011. Int Orthop 38: 1685-1690.

11. Lee YK, Ha YC, Park C (2013). Trends of surgical treatment in femoral neck fracture: a nationwide study based on claim registry. J Arthroplasty 28: 1839-1841.

12. National Institute for Health and Clinical Excellence (2011). NICE clinical guideline 124. Hip fracture: the management of hip fracture in adults. NICE

13. National Institute for Health and Clinical Excellence (2017) NICE clinical guideline 124 (addendum). Hip fracture: the management of hip fracture in adults. NICE

14. Walker LC, Lee LH, Webb M (2016) Provision of total hip replacement for displaced intracapsular hip fracture and the outcomes: an audit of local practice based on NICE guidelines. Hip Int 26: 153-7.

15. Lewis DP, Waever D, Thorninger R (2019) Hemiarthroplasty vs Total hip arthroplasty for the management of displaced neck of femur fractures: a systematic review and meta-analysis. J Arthroplasty; 34:1837-1843.

16. Jameson SS, Lees D, James P (2013) Cemented hemiarthroplasty or hip replacement for intracapsular neck of femur fracture? A comparison of 7732 matched patients using national data. Injury 44: 1940-44.

17. Van den Bekerom MP, Hilverdink EF, Sierevelt IN (2010) A comparison of hemiarthroplasty with total hip replacement for displaced intracapsular fracture of the femoral neck: a randomised controlled multicentre trial in patients aged 70 years and over. J Bone Joint Surg Br 92B: 1422-8.
18. Learmonth ID, Young C, Rorabeck C (2007) The operation of the century: total hip replacement. Lancet 370: 1508-1519.
Citation:

Morris R, Lewis MH, Bailey DM, Lewis PM (2019) Hemiarthroplasty or Total Hip Replacement for intracapsular Hip Fractures? A Dilemma in Trauma Surgery. Integr J Orthop Traumatol Volume 2 (5): 1-2. 